\title{
Implementation of District Health Information Software 2 (DHIS2) in Sri Lanka
}

Dr. Subodha Manoj MBBS, MSc

Medical Officer (Health Information), Ministry of Health, Sri Lanka

E-Mail address: subodha.manoj@gmail.com

Dr. Arjuna Wijekoon MBBS, MSc

Deputy Director (Health Information), Provincial Department of Health Services - Northwestern Province, Sri Lanka

E-Mail address: aawijekoon@gmail.com

Dr. Manjula Dharmawardhana MBBS, MSc

Medical Officer (Health Informatics), Ministry of Health, Sri Lanka

E-Mail address: manjulapra@gmail.com

Dr. Deepal Wijesuriya MBBS, MSc, MBA

Medical Officer (Health Informatics), National Institute of Health Sciences, Kalutara, Sri Lanka

E-Mail address: deepal.wijesooriya @gmail.com

Dr. Shan Rodrigo MBBS, MSc

Medical Officer (Health Informatics), National Programme for Tuberculosis Control and Chest Diseases, Ministry of Health, Sri Lanka

E-Mail address: semuthurodrigo@gmail.com

\section{Dr. Roshan Hewapathirana MBBS, MSc, MIEEE, SCMAD}

Research Fellow, Department of Informatics, Faculty of Mathematics and Natural Sciences, University of Oslo, Norway

E-Mail address: roshanhewapathirana@ gmail.com

Dr. Pandula Siribaddana MBBS, PG Dip. (Med. Edu.)

Research Fellow, Department of Informatics, Faculty of Mathematics and Natural Sciences, University of Oslo, Norway

E-Mail address: pasiribaddana@yahoo.com

Dr. Thilina Gunasekera MBBS, MSc

Medical Officer (Health Informatics), Ministry of Health, Sri Lanka

E-mail address: agtilinagunasekara150@gmail.com

Prof. Vajira H. W. Dissanayake MBBS, PhD

Founder Chairperson and Member, Specialty Board in Biomedical Informatics, Postgraduate Institute of Medicine, University of Colombo and Professor, Department of Anatomy and Human Genetics Unit, Faculty of Medicine, University of Colombo, Sri Lanka

E-Mail address: vajirahwd@hotmail.com

Sri Lanka Journal of Bio-Medical Informatics 2012;3(4):109-114

doi: http://dx.doi.org/10.4038/sljbmi.v3i4.5431

\section{Abstract}

District Health Information Software (DHIS) 2 is a tool for collection, validation, analysis, and presentation of aggregate and transactional data, tailored (but not limited) to integrated health information management 
activities (http://www.dhis2.org). In this paper we describe the introduction of DHIS2 to Sri Lanka and share our views on challenges and opportunities.

Keywords - District Health information Software; DHIS 2; Sri Lanka

\section{DHIS2 Overview}

District Health Information Software 2 (DHIS2) is an open-source software initially developed by the Health Information Systems Programme (HISP) at the University of Oslo. Since then it has evolved into a globally distributed development strategy ${ }^{(1)}$. Today developers from many countries including Norway, India, Vietnam, Tanzania, Ireland take part in the ongoing developments of the DHIS 2 platform $^{(2)}$.

In general, DHIS 2 can be described as a "tool for collection, validation, analysis, and presentation of aggregate statistical data, tailored (but not limited) to integrated health information management activities"(3). The users of DHIS 2 platform can customise the web based software package according to their health information needs without having to know or learn a complex programming language. This was made possible because DHIS 2 is a generic tool based on an open meta-data model and a flexible user interface ${ }^{(4)}$.

According to the official DHIS 2 website (dhis2.org), the software has been adapted for national Health Information Systems (HIS) deployment in seven countries - Kenya, Tanzania, Uganda, Rwanda, Ghana, Liberia, and Bangladesh ${ }^{(5)}$. In addition, more than 20 countries, including Sri Lanka, have made use of DHIS 2 at sub-national, programme specific or at pilot levels.

Apart from being open source, the adaptability of DHIS 2 to the requirements of various countries has been supported by many different features integrated within the DHIS 2 platform. These features include, customised data entry, indicator defining, data visualising through various types of graphs, web based pivot tabling, integrated GIS module, meta-data importing and exporting, custom data quality checks, user access control, integrating messaging system and DHIS 2 mobile solutions. In various DHIS 2 based projects described in this paper, these features have been customised to suit the Sri Lankan setting by mostly Medical and Dental graduates who have completed or are following the Masters Course in Biomedical Informatics at the Postgraduate Institute of Medicine (PGIM), University of Colombo.

\section{DHIS2 implementation in Sri Lanka}

There have been several initiatives aimed at DHIS2 implementation in Sri Lanka. They are sumarised in the case studies below. They fall into two broad categories:

Firstly, DHIS2 was used by several of the M.Sc. graduates for their M.Sc. research projects. These implementations showed that it was feasible to use DHIS2 in the Sri Lankan setting with appropriate customisation.

Secondly DHIS2 was used in several projects in the Ministry of Health; both centrally and in the provinces. These projects showed that large scale DHIS2 implementation was feasible in Sri Lanka. These however, have had to be stopped or are progressing slowly due to various reasons. 
In 2012 the World Health Organisation (WHO) designated the National Institute of Health Sciences (NIHS) in Kalutara, a WHO collaborating center for public health. The NIHS is considering implementing DHIS2 and their interest may be an opportunity for future DHIS2 implementation in Sri Lanka (See case study 7).

\section{Case Studies}

\section{Case 1: Maternal and Child health Management Information System}

DHIS2 was customised to manage Maternal and Child Health $(\mathrm{MCH})$ data from the peripheral public health facilities of Medical Officer of Health (MOH) level to the central Family Health Bureau (FHB) the central agency responsible for Maternal and Child care in Sri Lanka. This was initiated as a student project under the supervision of Deputy Director, FHB and piloted in the North Western Province.

In phase I, DHIS2 was piloted in one $\mathrm{MOH}$ area of the North Western Province and in phase II, piloting was expanded to five more $\mathrm{MOH}$ areas with training of $\mathrm{MOH}$ staff. There was a MOU signed between HISP India and Health Informatics Society of Sri Lanka (HISSL) to support customisations. A Biomedical Informatics graduate was employed by the University of Oslo for these DHIS2 customisations. Further, it was planned to sign a MOU between University of Oslo and Provincial Department of Health Services, North Western Province, but did not materialise during the pilot phase. The pilot project spanned from 1 October 2011 to 30 April 2012. During the pilot project, the provincial health department was satisfied with the flexibility of DHIS2 as a health information management tool and requested to scale up DHIS2 piloting as a province-wide public health information system. The FHB requested that further development be halted to give time to plan and develop a comprehensive health information system (HIS) to cover all public health data managed under the FHB.

\section{Case 2: School Health}

Similar to the attempt made for MCH data, DHIS2 was customised for the school health data stream. School health data was collected from government school through $\mathrm{MOH}$ and converged to the FHB. The customisation of DHIS2 was initiated under the supervision of Director, School Health. This project was authorised to pilot in schools of a district in the Western province. However, the student who 'owned' the project was appointed as Medical Officer-Health Information to a different institution and the piloting never started. A new project drawing on the experiences of this failed effort was initiated in 2012 on a different software platform. It has now been successfully piloted and handed over to the Ministry of Education for implementation.

\section{Case 3: A web-based Health Information database incorporating geographic information system (GIS)}

This project focused on mapping of health institutions under the Department of Health. All the health institutions were added to DHIS2 using the geo-coordinates given by WHO and the Japanese Agency for International Corporation.

\section{Case 4: A web-based system for management of health manpower information}

The health manpower information management project was coupled with the GIS mapping of health facilities mentioned in case three and focused on collecting health manpower information from health institutions. Piloting was planned in the Colombo District. After the initial customisation of the system in May 2012, piloting started at two base hospitals. Initial training was done and the staff initiated data entry. Progress of the system was halted due to 
lack of funding for hiring people and non-allocation of Department of Health staff for the project for data entry and supervision. A new project drawing on the experience of this failed effort was initiated in 2012 is now being successfully implemented in stages using a different software platform.

\section{Case 5: Health institution performance and facility information system}

This project focused on collecting, analysis and geographic representation of hospital services data, available facilities and human resources and financial information. The system was implemented in 13 hospitals that are directly under the control of the Ministry of Health which have Medical Officers in Health Informatics from July 2012. Medical Officers in Health Informatics at these institutions manage the programme locally and supervise the data entry and analysis within the scope of each institution. The Ministry of Health has now decided to support and expand this project.

\section{Case 6: DHIS2 for integrated e-registry for respiratory diseases}

The National Programme of Tuberculosis Control and Chest Diseases (NPTCCD) had the need for an integrated electronic registry to manage respiratory diseases and DHIS2 tracker was identified as a possible tool for computerising the respiratory diseases registries and aggregated patient information. The project was started with the support from ICT Agency (ICTA) of Sri Lanka and University of Kelaniya (UoK) for technical assistance for coding support as and when necessary. Health Information Unit (HIU) of the Ministry of Health provided a server to host the customised DHIS2 instance. Intel Sri Lanka is willing to consider funding the project in the initial phase with computers for the pilot stations and the first phase of piloting will be initiated in mid January 2013 in selected peripheral respiratory clinics and NPTCCD. ICTA and UoK agreed to explore funds collaboratively with the NPTCCD for the sustainability of the project after the phase I piloting. Discussions are going on for creating a DHIS2 instance based on generic requirements of all public health registries, under the guardianship of ICTA that can be used by any vertical public health programme to manage extended disease registries and programme information. It is expected to use GIS and facility data from previous DHIS2 instances to expedite the deployment of this DHIS2 instance.

\section{Case 7: DHIS2 at the National Institute of Health Sciences (NIHS) - the WHO Collaboration Center for Training in Public Health: An opportunity}

The NIHS is the premier training institute of the Ministry of Health for training of health manpower for Primary Health Care (PHC) programmes in the entire country. The main training groups are Public Health Midwives (PHM), Public Health Inspectors (PHI), Public Health Nurses (PHN), and Medical Officers of Health (MOH). NIHS is a WHO collaborating center and it is controlled directly under the Ministry of Health. It has two Medical Officers of Health areas as field practice areas - MOH Kalutara and MOH Beruwela. NIHS is fairly independent from other authorities though it works very closely with other institutions such as the Family Health Bureau. NIHS staff is an influential body in most of the public health decision making forums at national level.

The NIHS is looking for a data aggregating solutions for NIHS internal needs to use in NIHS field practice areas. If it is a good solution they will incorporate it into the public health curriculum of PHIs and PHMs. The NIHS has several Medical Offices in Health Informatics posted there who can take leadership in this efort. The NIHS has examined the solution 
piloted in the North Western Province (Case Study 1) and is happy to implement it, if appropriate support is provided.

\section{Challenges and recommendations}

The challenges for DHIS2 implementation in Sri Lanka and recommendations for overcoming them are given below:

1. "Political Leadership" - It is essential to obtain highest level endorsement for DHIS2 implementation at National (Ministry of Health) and Provincial Level and to communicate such endorsement to everyone concerned and have the agreement of all stakeholders in writing prior to starting any project.

2. "Project planning and financing" - It is necessary to identify needs for DHIS2 implementation, plan the projects (including identifying the project team and indicating their roles clearly), identify financial requirements and projects to the annual plan of the Ministry of Health and provincial ministries so that the projects are institutionalised and supported financially and otherwise. In the future, funding would become available for implementation of ICT based solutions in the health care system of Sri Lanka under a soft loan provided by the World Bank to the government of Sri Lanka.

3. "Development of a DHIS2 support team" - It is necessary to have a team of software developers who can customise DHIS2 to local needs as and when required. When an institution develops a project under the guidance of a Medical Officer or Dental Surgeon in Health Informatics and decide that it is implemented on a DHIS2 platform, there should be a team who can move in and do that work. This team can be a Ministry of Health Team. It is also possible to have that team in the proposed Health Informatics Unit to be set up in the Faculty of Medicine, University of Colombo. This can be done in collaboration with the University of Oslo and HISP India.

4. "Change Management" - It is necessary to ensure engagement of all stake holders and appropriate training of users of new systems to ensure that they are comfortable with what is being done. In a meeting of middle level manages of the health system in Sri Lanka in early 2012, lack of understanding by higher officials was seen as the biggest barrier for implementation of ICT salutations in the health system of Sri Lanka. It is heartning to note that this situation is fast changing.

\section{Conclusion}

DHIS2 was introduced to Sri Lanka through the M.Sc. in Biomedical Informatics course conducted by the Postgraduate Institute of Medicine of the University of Colombo in collaboration with the University of Oslo, Norway, with funding from Norad managed by the Norwegian Center for International Collaboration in Higher Education.

The graduates from this course, numbering 57 so far (with over 30 in training), have been designated as Medical Officers and Dental Surgeons in Health Informatics and appointed to various institutions in the health sector in Sri Lanka.

They have become a strong network for health information systems implementation in Sri Lanka. The Norad funding available since 2008 will come to an end in December 2013. The 
course however has now become sustainable and would continue to produce graduates in the future. In fact there are plans to introduce a Doctor of Medicine (MD) programme in Biomedical Informatics thus enabling the graduates to get further training in the field and become specialists in Health and Medical Informatics. These M.Sc. and MD graduates who have been exposed to DHIS2 would be the backbone for DHIS2 implementation in Sri Lanka.

\section{References}

1. Braa J, Muquinge M. Building collaborative networks in Africa on health information systems and open source software development-experiences from the HISP/BEANISH Network. IST Africa 2007:3

2. DHIS 2 is developed and used in a global basis: http://dhis2.org/node/6, Accessed on: 21.3.2013

3. Braa J, Kanter AS, Lesh N, Crichton R, Jolliffe B. et al. "Comprehensive yet scalable health information systems for low resource settings: a collaborative effort in Sierra Leone." AMIA Annual Symposium Proceedings. American Medical Informatics Association 2010: 372-76.

4. DHIS 2 is used worldwide: http://dhis2.org/node/30, Accessed on: 21.3.2013. 